\title{
A Theory of Participation in Elections*
}

\author{
Timothy J. Feddersen ${ }^{\dagger} \quad$ Alvaro Sandroni ${ }^{\ddagger}$
}

April 2002

\begin{abstract}
In this paper, we relax one of the central assumptions in game theory: that agents' payoffs are exogenously determined by the outcomes of the game. We develop a methodology in which agents' payoffs in a game are partly determined by endogenously generated preferences over actions. In order to predict outcomes we develop a solution concept we call consistency linking agents' preferences with actual behavior in a manner analogous to Nash equilibrium. We use this methodology to analyze a model of participation in elections in which voting is costly and no vote is ever pivotal. The resulting model delivers high turnout and comparative statics that are consistent with strategic behaviour.
\end{abstract}

${ }^{*}$ We thank many audiences for their helpful comments.

${ }^{\dagger}$ MEDS Department, Kellogg Graduate School of Management, 2001 Sheridan Road Evanston IL 60208 tfed@nwu.edu

‡Department of Economics, University of Rochester, 238 Harkness Hall Rochester NY 14627 alsn@troi.cc.rochester.edu and MEDS Department, Kellogg Graduate School of Management, 2001 Sheridan Road Evanston IL 60208. Sandroni thankfully acknowledge financial support from the National Science Foundation. 


\section{Introduction}

Behavior in laboratory experiments and in the field does not always correspond to the predictions of decision and game theory. Economists, game theorists, psychologists and political scientists have considered a variety of alternative approaches to explain these anomalies (see, for example, Simon (1955), Kahnemann and Tversky (1979), Becker (1976), Rabin (1993)). In this paper, we relax one of the central assumptions in game theory: that agents' payoffs are exogenously determined by the outcomes of the game. We develop a model in which agents' payoffs are also determined by endogenously generated preferences over actions. Agents' preferences over actions depend upon how they believe they should behave given the behavior of other agents. Because agents are not playing a standard game, existing solution concepts do not readily apply. We develop a solution concept we call consistency that links agents' preferences with actual behavior in a manner analogous to Nash equilibrium.

Rather than developing a general model our focus here is on demonstrating some of the basic features of our approach in the context of a large election. We have chosen to analyze participation in large elections because this is a partic-

ularly difficult problem for standard game-theoretic models. If voting is costly then, since the likelihood of a vote being pivotal is very small, standard gametheoretic models predict low levels of turnout (e.g., Palfrey and Rosenthal (1985)). Typically, we observe high levels of turnout. The discrepancy between theory and prediction in the case of turnout is taken by some as evidence against the applicability of rational choice theory to politics (see for example, Green and Shapiro (1996)). But a claim that voters are not instrumentally rational runs afoul of 
considerable evidence of strategic behavior by voters. For example, turnout levels are correlated with the expected closeness of the election (Blais (2000)); small increases in costs to vote can have significant impact on turnout levels (Riker and Ordeshook (1968)); and behavior in multicandidate elections shows compelling evidence of strategic voting (Franklin, Niemi and Whitten (1994)). The evidence for strategic behavior by voters is as puzzling as the fact of large scale turnout and for the same reason. Not only is the incentive to vote minimal but so is the incentive to vote strategically. The challenge is to provide a model that not only explains participation but also explains the comparative statics that seem to be consistent with strategic behavior.

Our approach is related to earlier work by Harsanyi. Harsanyi (1992) assumes that a fraction of the population are "rule-utilitarians." A rule-utilitarian is an agent who receives a payoff for acting according to a strategy profile (a rule) that, if taken by all agents, maximizes social welfare. In an earlier paper, Harsanyi (1977) even provides an example in which rule-utilitarians are motivated to vote. In his example there are two candidates, one of whom is assumed to maximize social welfare if elected. A critical assumption is that a fixed fraction of the population are voting for the socially inferior candidate. Harsanyi argues that rule-utilitarians will vote at a level to ensure the victory of the superior candidate. However, he provides no explanation for why some are voting for the inferior candidate. The rule-utilitarian model is problematic because if everyone agreed on the social welfare function then the inferior candidate would receive no votes. In that case the superior candidate may be elected with just one vote thereby minimizing the social cost of voting. ${ }^{1}$

\footnotetext{
${ }^{1}$ See Margolis (1982) for a less-formal ethics based model of participation.
} 
We model a two-candidate election in which a large population of agents may vote for candidate 1 , vote for candidate 2 or abstain. Voting costs vary within the population and a single vote is never pivotal. The winner of the election is the candidate that receives the majority of votes cast. Like Harsanyi, we assume that agents care about how they should behave. We assume that agents have preferences over the candidates and the cost of the election, but these preferences are not necessarily related to social welfare. Unlike Harsanyi, we allow for heterogenous preferences. There are two types of agents in our model: those who prefer candidate 1 (type 1) and those who prefer candidate 2 (type 2). The proportion of agents of each type is uncertain. Fixing the probability of winning for each candidate, all agents prefer to minimize the cost of the election.

In our model each agent has an action he should take and receives an additional payoff from taking this action. Riker and Ordershook (1968) analyze a model of participation in which agents receive a "duty" payoff $D>0$ when they vote for their preferred candidate. In their model the action an agent should take is determined exogenously. In contrast, the action an agent should take is determined endogenously in our model. In particular, it may be (and often is) the case that some agents receive an additional payoff for voting while others receive a payoff for not voting.

Given a preference type, let a rule define how each agent should behave (i.e. a rule is an arbitrary mapping from voting costs to the action space). We say that agents who act as they should are "doing their part." Some agents receive a payoff $D>0$ for doing their part, but not all of them do their part. Only those for whom it is not too costly. Let a social outcome be a probability that candidate 1 is elected and an expected cost of the election. Given the behavior 
of agents with preference type $j \neq i$, agents with preference type $i$ evaluate each rule by the social outcome they produce. That is, agents rank rules according to their preferences. We say that a behavior profile is consistent if the behavior of each agent follows from the agent's preferred rule (i.e. the rule with maximum ranking).

We show that if agents' behavior is consistent then there exists a cut-off point for each type such that agents with voting costs below this threshold should (and will) vote for their favored candidate. Agents with voting costs above the threshold abstain. As noted above, it is possible that agents with voting costs below $D$ will abstain because they reason they should.

There exists a one-to-one correspondence between the cut-off points that define a consistent profile and a pure strategy equilibrium of a suitably defined twoplayer game that we call $2 \mathrm{PG}$. So, we use $2 \mathrm{PG}$ to find consistent behavior profiles. However, a mixed strategy equilibrium in $2 \mathrm{PG}$ cannot be mapped into a consistent behavioral profile. This creates a difficulty because, as is often the case in games, an equilibrium in pure strategies may not exist in $2 \mathrm{PG}$. We show that a pure strategy Nash equilibrium exists in $2 \mathrm{PG}$ for an interesting class of elections and is unique.

Endogenizing the actions that agents should take allows our model to deliver comparative statics on turnout, winning probabilities and margin of victory that differ from Riker and Ordeshook. For example, in Riker and Ordeshook turnout levels are fixed and independent of the relative size of the minority. In contrast, our model predicts that turnout will be higher among the minority than the majority, yet the majority's preferred candidate wins with probability greater than a half. When the size of the minority becomes arbitrarily small our model 
becomes like Harsanyi's in which there is essentially only one type of preference. In that case, we show that turnout goes to zero. This result shows the importance of heterogeneity of preferences as a factor in explaining turnout. It also demonstrates why Harsanyi's model will not be able to explain turnout.

In the model of Palfrey and Rosenthal (1985), turnout levels are small but there is a negative correlation between turnout and margin of victory. Their result follows from the fact that the probability of a pivotal vote increases as the margin of victory decreases. Turnout and margin of victory may also be negatively correlated in our model. However, this correlation is not related to changes in the probability a vote is pivotal. Our result follows from the fact that the cut-off points determining whether an agent should vote or abstain are endogenous. When the size of the minority is small, agents in the majority group can win the election with high probability even with low turnout. In a consistent profile only a small fraction of a large majority will participate, but this fraction is sufficiently large to ensure a high chance of victory. So, expected turnout will be small and expected margin of victory large. As the size of the minority increases, the preferred rule of the majority requires a larger turnout. When the group sizes are equal, the expected margin of victory is minimized and expected turnout maximized. However, the expected turnout and margin of victory need not be inversely related when other parameters of the model vary. For example, we show a positive relationship between expected turnout and margin of victory as expected voting cost changes.

This paper is organized as follows: In section 1.1 we discuss the related literature within political science and economics. In section 2, we present our model. In section 2.1, we explain some of our modelling choices. In section 3 , we show 
the relationship between a consistent behavior profile and a pure strategy equilibrium in $2 P G$. We present an illustration of the properties of the model using a simple example in section 4 . In section 5 , we show the existence and uniqueness of pure strategy equilibrium in a class of elections in $2 \mathrm{PG}$. In section 6 , we show the general properties of our model. In section 7, we show how our model relates to other models of turnout. We conclude the paper in section 8. Proofs are in appendixes $A$ and $B$. In appendix $C$, we provide some formal justification for some of our assumptions.

\subsection{Literature Review}

Extensive literature reviews of the theoretical and empirical literature can be found in Aldrich (1993), Palfrey and Rosenthal (1983, 1985), Feddersen and Pe-

sendorfer (1999) and, most recently, Blais (2000). Blais (2000) reports that a sense of duty plays an important role in the decision to vote for a large fraction of voters. He writes:

I conclude that for many people voting is not only a right, it is also a duty. And the belief that in a democracy every citizen should feel obliged to vote induces many people to vote in almost all elections. That sense of duty is not shared by everyone. It may vary from one country to another. It can also vary over time. Blais (2000, page 113).

There are a wide variety of formal models of turnout and the surveys mentioned above discuss the formal literature. The papers most closely related to our effort here are those by Riker and Ordershook (1968), Ulhaner (1989), Harsanyi (1992), 
Morton (1991), and Nalebuff and Shachar (1999). In section 7, we discuss how all these papers relate to our model.

There is also some overlap with models explaining voluntary contributions to public goods. ${ }^{2}$ Standard models typically predict contributions levels below what is observed. Altruism has been proposed as an explanation for these anomalies (see, among others, Hirschleifer (1985), Monroe (1994) and Sugden (1984)). However, in models in which the marginal effect of a contribution decreases as total contributions increases, a "crowding out" effect is predicted. Thus, in a large population predicted contributions will be low. The crowding out effect is particularly severe in elections. If all voters prefer the same candidate then a single vote will crowd out all others. Hence, simple altruism cannot explain observed contribution patterns. Contributions will be larger if, as assumed by Becker (1977) and Andreoni (1989), agents receive a "warm glow" payoff for contributing for the public good which is independent of the contribution of others. As in the Riker and Ordeshook (1968) paper, the warm glow effect is exogenous.

There is a warm glow aspect to our model. While we do not assume that agents receive a warm glow payoff for voting, agents do receive an additional payoff for doing their part. Samuelson (1993), Bernheim and Stark (1988), and Bergstrom (1995), among others, address the problem of how ethical preferences may arise as the consequence of evolutionary pressures.

\footnotetext{
${ }^{2}$ The crucial difference is that the optimal level of contributions for a public good may or may not be large whereas the social optimal level of turnout is necessarily small. This follows because in a large election, any result can be reproduced, at lower social cost, by taking a sufficiently representative sample.
} 


\section{The Model}

We model an election with two candidates, 1 and 2 . There is a continuum of agents who must choose one of three actions: vote for candidate 1, vote for 2 or abstain. Let $A$ be the set of these three actions. The election is decided by majority rule.

Each agent has a cost of voting that is a realization of a random variable $\tilde{c}$ distributed over $\left[c^{l}, c^{u}\right], 0 \leqslant c^{l}<c^{u}$ with a continuous strictly positive density function $f_{\tilde{c}}$ on $\left[c^{l}, c^{u}\right]$. The cost of voting can be thought of as, for example, the time spent in the process of voting. We assume that the disutility from voting is equal to the cost and that each agent's cost of voting is independent of any other random variable in this model. Each agent knows her own realized voting costs, but not the realization of other agents' voting costs.

There are two types of agents. Type 1 agents prefer candidate 1 and type 2 prefer candidate 2. All agents prefer the social cost of voting to be minimized. ${ }^{3}$ Formally, type 1 agents have a utility function given by

$$
w p-\vartheta(\phi)
$$

where $\vartheta:[0,1] \rightarrow \Re_{+}$is a strictly increasing, convex, twice differentiable function, $p$ is the probability candidate 1 wins the election, $\phi$ is the expected social cost of voting and $w \in \Re_{+}$is a parameter of the model that we call the importance of the

\footnotetext{
${ }^{3}$ The social cost of voting is given by

$$
\int_{c^{l}}^{c^{u}} c \beta(c) f_{\tilde{c}}(c) \partial c
$$

where $\beta(c)$ is the fraction of the population with costs $c$ that voted.
} 
election. ${ }^{4}$ Type 2 agents have an utility function given by

$$
w(1-p)-\vartheta(\phi)
$$

No single agent decides who will be elected or the social cost of voting. Each agent's preferences reflect the choices he would make if he was a social planner. More formally, let a lottery be a random variable that determines the elected candidate and the social cost of voting. We assume that if he had the opportunity, an agent type 1 (2) would choose among these lotteries according to $2.1(2.2) .^{5}$

We assume that the fraction of agents of each type is unknown. The fraction of type 1 agents within the population is given by a random variable $\tilde{k}$ with expected value $k$. The parameter $k$ may be thought of as indicating the level of disagreement within the population. When $k$ is small almost everyone in the population agrees that candidate 2 is preferred to candidate 1 . When $k$ is close to .5 the society is nearly divided on the question of which candidate is preferable.

The model as defined so far is fairly standard. It is known that in voting games, as above, with a continuum of agents and costly voting there are generically no equilibria in which a positive fraction of the population participates. We now alter this standard game. We assume that every agent has an action that he understands to be the action he should take (and some agents derive positive utility from acting as they should). As will become clear, agents endogenously determine the actions they should take as well as the actions they do take. Hence,

\footnotetext{
${ }^{4}$ Holding everything else constant, the higher the value of $w$ the higher the expected social cost that agents would be willing to trade for an increase in the chances that their favored candidate wins. Thus, $w$ parameterizes the importance of the difference between the two candidates relative to the social cost of voting.

${ }^{5}$ The assumption of two types is for simplicity only.
} 
our assumption differs in a fundamental way from an assumption that agents receive positive utility for taking some exogenously determined action, e.g., voting for one of the candidates. We now define these ideas formally.

We call a function $e_{i}:\left(c^{l}, c^{h}\right) \longrightarrow A, i \in\{1,2\}$, a rule. We say that if a rule $e_{i}$ is chosen by agents with preferences type $i \in\{1,2\}$, then $e_{i}(c)$ denotes the action an agent type $i$ with cost $c$ should take. As mentioned above, we say that agents who take the action they should are "doing their part." Some agents (called ethical agents) receive a payoff $D>c^{l}$ for doing their part. Other agents (called abstainers) receive zero payoff for doing their part. The fraction of ethical agents in group $i \in\{1,2\}$ is given by the random variable $\tilde{q}_{i}$ with expected value $q_{i}$.

The fact that an agent has an action that he should take does not guarantee that he will take it. If a rule $e_{i}$ is chosen by agents with preferences type $i \in\{1,2\}$ then $s_{i}^{e_{i}}:\left(c^{l}, c^{h}\right) \times\{0, D\} \longrightarrow A$ such that

$$
s_{i}^{e_{i}}(c, d)=\left\{\begin{array}{cr}
\text { abstain } & \\
e_{i}(c) & \text { if } \begin{array}{c}
d \leq c \\
\end{array}
\end{array}\right.
$$

denotes the action each type $i$ agent will take. ${ }^{6}$ That is, ethical agents will do their part if and only if the cost of the required action is sufficiently small. All others abstain.

We assume that agents' rank rules according to their preferences. Formally, let $s_{i}:\left(c^{l}, c^{h}\right) \times\{0, D\} \longrightarrow A$ be a behavioral profile for type $i \in\{1,2\}$ agents. Given a pair $\left(s_{1}, s_{2}\right)$ of behavioral profiles, let $p\left(s_{1}, s_{2}\right)$ be the probability that candidate 1 wins the election and $\phi\left(s_{1}, s_{2}\right)$ be the expected social voting cost. Type 1 agents

\footnotetext{
${ }^{6}$ The domain of a rule could also be $\left(c^{l}, c^{h}\right) \times\{0, D\}$. This would not change any results.
} 
rank rules as follows. Given a strategy profile $s_{2}$ for type 2 agents, the higher the value of $R_{1}\left(e \mid s_{2}\right)$

$$
R_{1}\left(e \mid s_{2}\right) \equiv w p\left(s_{1}^{e}, s_{2}\right)-\vartheta\left(\phi\left(s_{1}^{e}, s_{2}\right)\right)
$$

the higher agents with preferences 1 rank rule $e$. Analogously, given the strategy profile $s_{1}$, the higher the value of $R_{2}\left(e \mid s_{1}\right)$,

$$
R_{2}\left(e \mid s_{1}\right) \equiv w\left(1-p\left(s_{1}, s_{2}^{e}\right)\right)-\vartheta\left(\phi\left(s_{1}, s_{2}^{e}\right)\right)
$$

the higher agents with preferences 2 rank rule $e$. That is, rules are ranked according to the outcomes the rule produces, taking as given the actions of agents with different preferences.

We say that a behavior profile $\left(s_{1}^{*}, s_{2}^{*}\right)$ is consistent when each agent's action is determined by a rule that is maximally ranked for her type, given the behavior of other types.

Definition 1 ((Consistency Requirement)). The pair $\left(s_{1}^{*}, s_{2}^{*}\right)$ is a consistent behavior profile if there exists a pair of rules $\left(e_{1}^{*}, e_{2}^{*}\right)$ such that

1.

$$
\begin{aligned}
& R_{1}\left(e_{1}^{*} \mid s_{2}^{*}\right) \geqslant R_{1}\left(e \mid s_{2}^{*}\right) \text { for all rules } e:\left(c^{l}, c^{h}\right) \longrightarrow A ; \\
& R_{2}\left(e_{2}^{*} \mid s_{1}^{*}\right) \geqslant R_{2}\left(e \mid s_{1}^{*}\right) \text { for all rules } e:\left(c^{l}, c^{h}\right) \longrightarrow A
\end{aligned}
$$

2.

$$
s_{i}^{*}=s_{i}^{e_{i}^{*}}, i \in\{1,2\} .
$$

Part 1 of the definition ensures that all agents choose a rule that is maximally ranked given the behavior of the other types. Part 2 ensures that the behavior of all agents of the same type is determined by the same maximally ranked rule. 


\subsection{Comments on the Model}

In our model agents have preferences about which candidate wins and the social cost of turnout. These preferences can be determined by asking agents to decide separately on the probability that candidate 1 is elected and the social cost of the election. Informally, we have in mind that agents' preferences reflect not only their self-interest but also their religious, ethical or philosophical perspectives. For example, these preferences might be associated with a notion of social welfare (utilitarianism), a concern for distributive justice or support for human rights. ${ }^{7}$

We assume that, fixing the probability candidate 1 wins the election, all agents prefer lower turnout since it produces lower social costs. Readers may wonder if this assumption is at odds with the fact that so many people worry about low turnout. Expressions of concern about low turnout need not represent a concern for turnout per se. Instead, people may be concerned by what low turnout signals about society e.g., lack of civic mindedness. If the winner of the election is fixed (independently of the votes) along with, for example, the level of civic-mindedness then a desire to maximize turnout seems hard to justify. Even so, including agents who prefer high turnout (or are indifferent about turnout) is not problematic. We only require that some agents prefer to minimize social cost. We do not include agents that prefer high turnout in this paper because these agents vote if and only if their cost to vote is smaller than their payoff for doing their part. In effect, we would be assuming what we are trying to explain.

\footnotetext{
${ }^{7}$ See Roemer (1996) for an exposition of these ideas.
} 


\subsubsection{Ranking of Rules}

In the model section, we show that the ranking of rules is ultimately implied by the agents' preferences. Taking as given the behavior of types different than their own, type $i$ agents rank rules as if they had used the following thought experiment. Assume that a rule $e$ defines the actions that all type $i$ agents should take. The outcome associated with $e$ is the probability candidate $i$ wins and an expected social cost of voting. The ranking of rules corresponds to the ranking of associated outcomes as given by the preferences type $i$.

There are several implications of these rankings that require explanation. First, rules are ranked according to the outcome produced when they define the actions that should be taken. Consider the following alternative: agents rank rules according to the outcome that would be produced if all agents in their group were to act as they should under the rule. This alternative ranking would be written

$$
R_{1}\left(e \mid s_{2}\right) \equiv w p\left(e, s_{2}\right)-\vartheta\left(\phi\left(e, s_{2}\right)\right)
$$

This ranking might seem natural but it would generally make it impossible to find behavior profiles that satisfy our consistency requirement. A simple example illustrates the problem. Suppose that type 2 agents are all voting for candidate 2 and that the only way type 1 agents can win is if all such agents, including abstainers, vote for candidate 1 . Then, if rules were ranked under the assumption that every type 1 agent acts as they should, the best rule would require all such agents to vote for 1 because by so doing 1 will be elected. ${ }^{8}$ However, it is common knowledge among both types that 2 will be elected because abstainers (or ethical agents with voting costs higher than $D$ ) will not act as they should and vote for

\footnotetext{
${ }^{8}$ Here we assume that voting costs are sufficiently low.
} 
candidate 1. Instead they will abstain. Under this alternative ranking agents would be evaluating rules under premises they know to be false. This violates part 2 of our consistency definition. We avoid such contradictions by ensuring that when agents rank rules, they take into account agents' incentives for doing their part. Under our definition the best rule for agents type 1 in this example is for all of them to abstain.

We also assume that when agents rank rules they take as given the behavior of agents with preferences different from their own. In a note available upon request from the authors, we provide a formal foundation for this assumption. It will be helpful now to discuss informally what we might have done instead. At one extreme, we might have assumed an agent ranks rules taking as given the behavior of all other agents. In our model with a continuum of agents the act of an individual agent does not have any impact on either the probability a candidate wins or on the social cost of voting. So, the behavior of this agent can be exogenously determined. In suitably redefined model with countably many agents this agent would abstain. Since we already have a set of agents who abstain it would be redundant to include such types. At the other extreme, we might have assumed that some agents rank rules as if they define the actions everyone should take. A simple example will illustrate the problem with such an assumption. Under majority rule type 1 agents would rank as best a rule (applied to all agents) that would direct one agent (with $\operatorname{cost} c<D$ ) to vote for candidate 1 and everyone else to abstain. Type 1 agents would evaluate this rule under the assumption that everyone (including type 2 agents) accepts the rule and behaves accordingly. The result would be that candidate 1 wins the election at a social cost of 0 . Analogously, type 2 agents would rank as best a rule that directs one agent to 
vote for candidate 2 and everyone else to abstain. This clearly violates part 2 of our definition of consistency. That is, agents would evaluate rules assuming that everyone will accept the same rule when they know this will not be the case. In the note mentioned above, we consider a model in which each agent could, a priori, take as given the behavior of any group of agents. We show that in our two candidate model, consistency implies that we can assume, without loss of generality, that agents take as given the behavior of agents with preferences different from their own.

\section{2PG}

In this section, we show that there exists a one-to-one correspondence between a consistent behavior profile and a Nash-equilibrium, in pure strategies, of a suitably chosen two player game that we call $2 \mathrm{PG}$. The only role of $2 \mathrm{PG}$ is to simplify the computation of a consistent behavior profile.

Proposition 1. Let $\left(s_{1}^{*}, s_{2}^{*}\right)$ be a consistent behavior profile. There is a pair of cut-off points $c_{i}^{*} \in\left(c^{l}, D\right], i \in\{1,2\}$, such that for almost all costs $c \in\left[c^{l}, c^{u}\right]$;

$$
\begin{array}{cc}
s_{i}^{*}(c, D)=e_{i}^{*}(c)=\text { vote for } i & \text { if } c \in\left[c^{l}, c_{i}^{*}\right] \\
s_{i}^{*}(c, D)=e_{i}^{*}(c)=\text { abstain } & \text { if } c \in\left(c_{i}^{*}, c^{u}\right] .
\end{array}
$$

Proof: See Appendix A.

Proposition 1 shows that if $\left(s_{1}^{*}, s_{2}^{*}\right)$ is a consistent behavior profile then there exists a pair of cut-off points $\left(c_{1}^{*}, c_{2}^{*}\right) \in\left(c_{l}, D\right)$ such that type $i$ ethical agents should and will vote for $i$ when their voting cost is smaller than $c_{i}^{*}$. They also should and will abstain when their voting cost is greater than $c_{i}^{*}$. Therefore, there 
is no loss of generality in assuming that a behavior profile can be defined by a pair of cut-off points.

The intuition behind this result is that the least costly way to achieve a given level of turnout is to have low-cost agents vote and high-cost agents abstain. The fact that the cut-off point $c_{i}^{*}, i \in\{1,2\}$, is strictly less than $D$ follows from the fact that agents with costs above $D$ necessarily abstain.

We now determine which pairs of cut-off points define consistent behavior. Assume that type $i$ ethical agents vote if and only if their cost is below some cut-off point $c_{i} \leqslant D$. Then, the fraction of type $i$ ethical agents who vote is

$$
\sigma_{i}\left(c_{i}\right) \equiv \int_{c^{l}}^{c_{i}} f_{\tilde{c}}(x) \partial x .
$$

The maximum fraction of type $i$ ethical agents who vote is

$$
\sigma^{u} \equiv \int_{c^{l}}^{\min \left\{D, c^{u}\right\}} f_{\tilde{c}}(x) \partial x .
$$

If a fraction $\sigma_{i}$ of type $i$ ethical agents vote, the expected social cost of voting associated with this behavior is

$$
C_{i}\left(\sigma_{i}\right) \equiv k_{i} q_{i} \int_{c^{l}}^{c_{i}\left(\sigma_{i}\right)} f_{\tilde{c}}(x) x \partial x .
$$

where $c_{i}\left(\sigma_{i}\right)$ is the inverse function of $\sigma_{i}\left(c_{i}\right)$. The expected social cost of voting is $C_{1}\left(\sigma_{1}\right)+C_{2}\left(\sigma_{2}\right)$. Candidate 1 wins the election if he gets the most votes. Hence, under the assumption that, for $i=1,2$, a fraction $\sigma_{i}>0$ of type $i$ ethical agents vote (for $i$ ), candidate 1 wins if

$$
\tilde{k} \tilde{q}_{1} \sigma_{1} \geqslant(1-\tilde{k}) \tilde{q}_{2} \sigma_{2} \Leftrightarrow \frac{(1-\tilde{k}) \tilde{q}_{2}}{\tilde{k} \tilde{q}_{1}} \leqslant \frac{\sigma_{1}}{\sigma_{2}} .
$$

So, candidate 1 wins the election with probability

$$
F_{\frac{(1-\tilde{k}) \tilde{q}_{2}}{\tilde{k} \tilde{q}_{1}}}\left(\frac{\sigma_{1}}{\sigma_{2}}\right),
$$


where $F_{\tilde{x}}(x) \equiv P(\tilde{x} \leqslant x)$ is the cumulative distribution function of a random variable $\tilde{x}$.

Analogously, candidate 2 wins the election with probability

$$
F_{\frac{\tilde{k} \tilde{q}_{1}}{(1-\hat{k}) \tilde{q}_{2}}}\left(\frac{\sigma_{2}}{\sigma_{1}}\right) .
$$

We now define 2PG. To avoid confusion, we call the decision makers in 2PG players while the voters in our underlying model are agents or types. Players do not represent anyone in the model. As mentioned above, $2 \mathrm{PG}$ is merely a device used to compute consistent behavior profiles.

A pure strategy for player $i \in\{1,2\}$ is a real number $\sigma_{i} \in\left(0, \sigma^{u}\right]$. The payoff for player 1 is given by

$$
\Pi_{1}\left(\sigma_{1} \mid \sigma_{2}\right) \equiv w F_{\frac{(1-\tilde{k}) \tilde{q}_{2}}{\tilde{\bar{k}}}}\left(\frac{\sigma_{1}}{\sigma_{2}}\right)-\vartheta\left(C_{1}\left(\sigma_{1}\right)+C_{2}\left(\sigma_{2}\right)\right) .
$$

The payoff for player 2 is given by

$$
\Pi_{2}\left(\sigma_{2} \mid \sigma_{1}\right) \equiv w F_{\frac{\tilde{k} \tilde{q}_{1}}{(1-\bar{k}) \tilde{q}_{2}}}\left(\frac{\sigma_{2}}{\sigma_{1}}\right)-\vartheta\left(C_{1}\left(\sigma_{1}\right)+C_{2}\left(\sigma_{2}\right)\right) .
$$

The pair $\left(\sigma_{1}^{*}, \sigma_{2}^{*}\right)$ is an equilibrium if

$$
\sigma_{1}^{*}=\arg \max _{\sigma_{1} \in\left(0, \sigma_{1}^{u}\right]} \Pi_{1}\left(\sigma_{1} \mid \sigma_{2}^{*}\right) \text { and } \sigma_{2}^{*}=\arg \max _{\sigma_{2} \in\left(0, \sigma_{2}^{u}\right]} \Pi_{2}\left(\sigma_{2} \mid \sigma_{1}^{*}\right) \text {. }
$$

Proposition 2. A pair of cut-points $\left(c_{1}^{*}, c_{2}^{*}\right)$ defines consistent behavior if and only if the pair $\left(\sigma_{1}^{*}, \sigma_{2}^{*}\right), \sigma_{i}^{*}=\sigma_{i}\left(c_{i}^{*}\right), i \in\{1,2\}$, is an equilibrium in $2 P G$.

Proposition 2 shows that $2 \mathrm{PG}$ can be used to obtain a consistent behavior profile. Given an equilibrium $\left(\sigma_{1}^{*}, \sigma_{2}^{*}\right)$ of $2 \mathrm{PG}$, the pair of cut-off points $\left(c_{1}^{*}, c_{2}^{*}\right)=$ $\left(c_{1}\left(\sigma_{1}^{*}\right), c_{2}\left(\sigma_{2}^{*}\right)\right)$ defines consistent behavior. Conversely, any pair of cut-off points $\left(c_{1}^{*}, c_{2}^{*}\right)$ that defines consistent behavior can be obtained by an equilibrium $\left(\sigma_{1}^{*}, \sigma_{2}^{*}\right)$ of 2 PG. Proposition 2 follows directly from the definitions above. Therefore, we omit the proof. 


\section{Main Results}

In this section, we analyze a simple example in which an equilibrium exists and is unique.

Example 1. The fraction of ethical agents in each group, $\widetilde{q}_{1}$ and $\widetilde{q}_{2}$, are independent and uniformly distributed over $[0,1]$. The fraction of the population in group 1 is deterministic and given by $k \in(0,1 / 2] .{ }^{9}$ So, group 1 is a minority. The costs of voting for each individual is given by $\frac{\bar{c}}{2}>0$ multiplied by an independent random variable that is uniformly distributed over the interval $(0,1)$. The social cost function is given by $\vartheta(x)=x$.

In this example, the cost of voting is given by

$$
C_{i}\left(\sigma_{i}\right)=\frac{1}{2} k_{i} \int_{0}^{\sigma_{i}} \frac{\bar{c}}{2} x \partial x=k_{i}\left(\frac{\bar{c}}{4}\right)\left(\sigma_{i}\right)^{2}
$$

Let $\bar{F}$ be the cumulative distribution function of $\frac{\widetilde{q}_{1}}{\widetilde{q}_{2}}$ (and of $\frac{\widetilde{q}_{2}}{\widetilde{q}_{1}}$ ). Let $f$ be the density function of $\frac{\widetilde{q}_{1}}{\widetilde{q}_{2}}$ and $\frac{\widetilde{q}_{2}}{\widetilde{q}_{1}}$. These functions are given by

$$
\begin{aligned}
& \bar{F}(z)=\frac{z}{2} \quad \text { if } z \leqslant 1 ; \quad f(z)=\frac{1}{2} \quad \text { if } z \leqslant 1 \text {; } \\
& \bar{F}(z)=1-\frac{1}{2 z} \text { if } z \geqslant 1 . \quad f(z)=\frac{1}{2 z^{2}} \quad \text { if } z \geqslant 1 .
\end{aligned}
$$

We can now define $2 \mathrm{PG}$ in this example. The payoff for player $i \in\{1,2\}$ is

$$
\Pi_{i}\left(\sigma_{i} \mid \sigma_{j}\right)=w \bar{F}\left(\frac{\sigma_{i} k_{i}}{\sigma_{j}\left(1-k_{i}\right)}\right)-k_{i}\left(\frac{\bar{c}}{4}\right)\left(\sigma_{i}\right)^{2}-k_{j}\left(\frac{\bar{c}}{4}\right)\left(\sigma_{j}\right)^{2} .
$$

Player $i$ optimize $\Pi_{i}\left(\sigma_{i} \mid \sigma_{j}\right)$ subject to $0<\sigma_{i} \leqslant \sigma^{u}=\min \left\{\frac{2 D}{\bar{c}}, 1\right\}$.

\footnotetext{
${ }^{9}$ To ease the notation, we define $k_{1} \equiv k$ and $k_{2} \equiv 1-k$.
} 


\subsection{The equilibrium}

Consider first the case that the payoff for doing their part is greater than any realized voting cost (i.e., $\left.D>\frac{\bar{c}}{2}\right)$. The constraint for each player is $(0,1]$. Let $\bar{k} \equiv \frac{k}{1-k}$. The first order conditions of the maximization problem for player 1 are:

$$
w f\left(\bar{k} \frac{\sigma_{1}}{\sigma_{2}}\right) \bar{k} \frac{1}{\sigma_{2}}-k\left(\frac{\bar{c}}{2}\right) \sigma_{1}\left\{\begin{array}{llc}
=0 & \text { if } & \sigma_{1} \in(0,1) \\
\geq 0 & \text { if } & \sigma_{1}=1 .
\end{array}\right.
$$

Note that $f(z)=f\left(\frac{1}{z}\right) \frac{1}{z^{2}}$. So, the first order conditions for player 2 can be rewritten as

$$
w f\left(\bar{k} \frac{\sigma_{1}}{\sigma_{2}}\right) \frac{\bar{k} \sigma_{1}}{\left(\sigma_{2}\right)^{2}}-(1-k)\left(\frac{\bar{c}}{2}\right) \sigma_{2}\left\{\begin{array}{ccc}
=0 & \text { if } & \sigma_{2} \in(0,1) \\
\geq 0 & \text { if } & \sigma_{2}=1
\end{array}\right.
$$

Assume that $\sigma_{2}=1$ and $\sigma_{1}<1$ is an equilibrium. Then, the first order conditions imply the following contradiction:

$$
k\left(\frac{\bar{c}}{2}\right) \sigma_{1}=w f\left(\bar{k} \sigma_{1}\right) \bar{k}>w f\left(\bar{k} \sigma_{1}\right) \bar{k} \sigma_{1} \geqslant(1-k)\left(\frac{\bar{c}}{2}\right)>k\left(\frac{\bar{c}}{2}\right) \sigma_{1}
$$

There are three types of equilibria to consider: an interior equilibrium $\left(\sigma_{1}<1\right.$ and $\left.\sigma_{2}<1\right)$; a corner solution $\left(\sigma_{1}=1\right.$ and $\left.\sigma_{2}=1\right)$; and a semi-corner solution $\left(\sigma_{1}=1\right.$ and $\left.\sigma_{2}<1\right)$.

We first look for an interior equilibrium. Dividing the first order conditions for 1 by condition for 2 yields $\frac{\sigma_{1}}{\sigma_{2}}=\sqrt{\frac{1}{k}}$. This implies that the expected turnout of the majority is higher than the minority (i.e, $\bar{k} \frac{\sigma_{1}}{\sigma_{2}}<1$ ) and, hence, $f\left(\bar{k} \frac{\sigma_{1}}{\sigma_{2}}\right)=0.5$. This simplifies the first order conditions and gives an unique solution

$$
\sigma_{1}^{*}=\sqrt{\frac{w}{\bar{c}}} \frac{1}{\sqrt[4]{k(1-k)}} \text { and } \sigma_{2}^{*}=\sqrt{\frac{w}{\bar{c}}} \sqrt[4]{\frac{k}{(1-k)^{3}}}
$$


The requirement that $\sigma_{1}^{*}<1$ and $\sigma_{2}^{*}<1$ implies

$$
\frac{\bar{c}}{w}>\frac{1}{\sqrt{k(1-k)}} .
$$

So, an interior equilibrium only exists if the expected cost to vote (relative to the importance of the election) is sufficiently high. If this restriction is not satisfied we either have a semi-corner or a corner solution.

A semi-corner equilibrium exists and is unique when the expected cost to vote (relative to the importance of the election) is at an intermediary level, satisfying

$$
\frac{k}{(1-k)^{2}}<\frac{\bar{c}}{w} \leqslant \frac{1}{\sqrt{k(1-k)}}
$$

To see this note that, in a semi-corner equilibrium, dividing the first order conditions for 1 by the condition for 2 yields $\sigma_{2} \geqslant \sqrt{\bar{k}}$. So, $f\left(\bar{k} \frac{1}{\sigma_{2}}\right)=0.5$. Hence, the unique solution is

$$
\sigma_{1}^{*}=1 \text { and } \sigma_{2}^{*}=\sqrt[3]{\frac{w k}{\bar{c}(1-k)^{2}}} .
$$

A corner solution is an equilibrium if and only if $w f(\bar{k}) \bar{k} \geqslant k\left(\frac{\bar{c}}{2}\right)$ and $w f(\bar{k}) \bar{k} \geqslant(1-k)\left(\frac{\bar{c}}{2}\right)$. However, $f(\bar{k})=0.5$. Therefore, $\sigma_{1}^{*}=\sigma_{2}^{*}=1$ is an equilibrium if and only if $\frac{\bar{c}}{w} \leq \frac{k}{(1-k)^{2}}$.

The equilibrium will be corner, semi-corner or interior depending on whether the expected cost of voting (relative to the importance of the election) is low, intermediate, or high, respectively.

The equilibrium for the case $D \leqslant \frac{\bar{c}}{2}$ can be obtained in an analogous way as the computation conducted above for the case $D>\frac{\bar{c}}{2}$. We, therefore, omit the algebra and report only the results. The equilibria for this case are displayed in 
Table 1.

Table 1. Equilbria for $D \leq \frac{\bar{c}}{2}$

\begin{tabular}{|l|l|l|}
\hline$\sigma_{1}^{*}=\sqrt{\frac{w}{\bar{c}}} \frac{1}{\sqrt[4]{k(1-k)}}, \sigma_{2}^{*}=\sqrt{\frac{w}{\bar{c}}} \sqrt[4]{\frac{k}{(1-k)^{3}}}$ & if & $\sqrt{\frac{w}{\bar{c}}} \frac{1}{\sqrt[4]{k(1-k)}}<\frac{D}{2 \bar{c}}$ \\
\hline$\sigma_{1}^{*}=\frac{D}{2 \bar{c}}, \sigma_{2}^{*}=\sqrt[3]{\frac{w k D}{2 \bar{c}^{2}(1-k)^{2}}}$ & if & $\sqrt[3]{\frac{w k D}{2 \bar{c}^{2}(1-k)^{2}}}<\frac{D}{2 \bar{c}} \leqslant \sqrt{\frac{w}{\bar{c}}} \frac{1}{\sqrt[4]{k(1-k)}}$ \\
\hline$\sigma_{1}^{*}=\sigma_{2}^{*}=\frac{D}{2 \bar{c}}$ & if & $\frac{D}{2 \bar{c}} \leqslant \sqrt[3]{\frac{w k D}{2 \bar{c}^{2}(1-k)^{2}}}$. \\
\hline
\end{tabular}

\subsection{Properties of the equilibrium}

We now illustrate the properties of our example with respect to expected turnout, expected margin of victory and the probability of victory of the majority. In equilibrium, expected turnout is

$$
T \equiv E\left(k \sigma_{1}^{*} \widetilde{q}_{1}+(1-k) \sigma_{2}^{*} \widetilde{q}_{2}\right)=0.5\left(k \sigma_{1}^{*}+(1-k) \sigma_{2}^{*}\right)
$$

The expected margin of victory is:

$$
\begin{gathered}
M V \equiv E\left|\frac{(1-k) \widetilde{q}_{2} \sigma_{2}^{*}-k \widetilde{q}_{1} \sigma_{1}^{*}}{(1-k) \widetilde{q}_{2} \sigma_{2}^{*}+k \widetilde{q}_{1} \sigma_{1}^{*}}\right|= \\
\frac{k \sigma_{1}^{*}}{(1-k) \sigma_{2}^{*}}\left(2 \ln 2-1-\ln \left(1+\frac{(1-k) \sigma_{2}^{*}}{k \sigma_{1}^{*}}\right)\right)+\frac{(1-k) \sigma_{2}^{*}}{k \sigma_{1}^{*}} \ln \left(1+\frac{k \sigma_{1}^{*}}{(1-k) \sigma_{2}^{*}}\right) .
\end{gathered}
$$

The probability of victory for candidate 2 (supported by the majority) is:

$$
P V \equiv \bar{F}\left(\frac{\sigma_{2}^{*}(1-k)}{\sigma_{1}^{*} k}\right)
$$

The expected margin of victory and the probability of victory for candidate 2 are increasing functions of $\frac{(1-k) \sigma_{2}^{*}}{k \sigma_{1}^{*}} \geqslant 1$. Hence, the comparative statics results for the expected margin of victory and the probability of victory for candidate 2 are identical. The equilibrium is described in closed-from solutions in section 4.1. Hence, $T, M V$ and $P V$ can be easily obtained as a function of the parameters 
$(w, \bar{c}, k, D)$. The properties of the equilibrium (presented below) are obtained by direct investigation of these functions..

Property 1. The expected fraction of agents in the majority group who vote $\left(0.5 \sigma_{2}^{*}\right)$ is smaller than the expected fraction of agents in the minority group who vote $\left(0.5 \sigma_{1}^{*}\right)$. However, the total expected turnout of the majority $\left(0.5(1-k) \sigma_{2}^{*}\right)$ is greater than the total expected turnout of the minority $\left(0.5 k \sigma_{1}^{*}\right)$.

Figure 1 below illustrates property 1.

Figure 1. Expected fraction of turnout in each group as a function of the level of disagreement $(\mathrm{k})$.

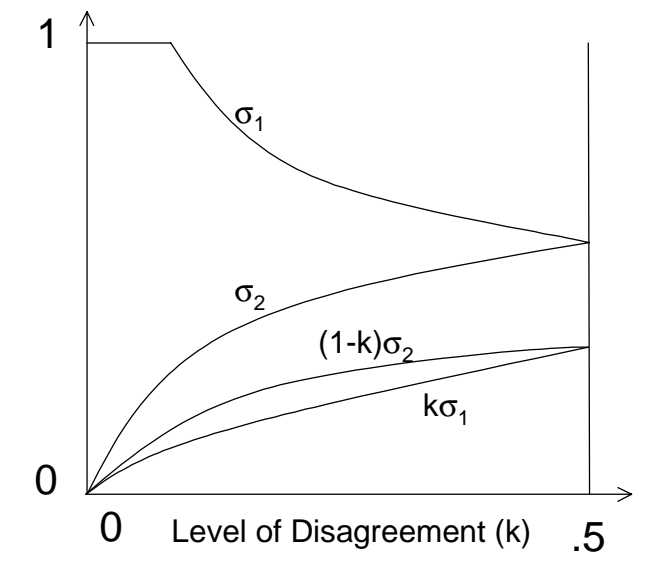

The figure holds for the case in which $D$ is greater than $\bar{c} / 2$ and $\bar{c} / w>2$.

The higher turnout of the minority follows from the fact that while the importance of the election is identical for both types the voting cost of a fraction of the minority is smaller then the voting cost of the same fraction of the majority. The fact that the candidate of the majority has a high probability of victory 
also depends upon the assumption that the importance of the election and the expected fraction of ethicals are the same for both groups. If the importance of the election were higher for agents in the minority group (or the minority had higher expected fraction of ethicals) then their favored candidate could win the election with probability greater than 0.5 .

Property 2. Expected turnout is strictly positive, and converges to zero as the level of disagreement goes to zero.

Property 2 shows that turnout is not simply a consequence of our assumption that some agents receive positive payoff for doing their part. It also depends upon the level of disagreement in the society. The intuition for property 2 is that as group 2 becomes an overwhelming majority they can vote at a low level and win the election with high probability.

Property 3. Expected turnout is increasing in the level of disagreement while the expected margin of victory is decreasing in the level of disagreement.

Property 3 illustrates how our model can produce an inverse correlation between margin of victory and turnout that has often been the focus of study in the empirical literature (see Matsusaka (1991), Nalebuff and Shachar (1999) and Blais (2000)). Since a vote is never pivotal in our setting, the relationship between turnout and margin of victory is unrelated to changes in the likelihood a vote is pivotal. Properties 2 and 3 are illustrated in Figure 2 below. 
Figure 2. Expected margin of victory and turnout as a function of the level of disagreement.

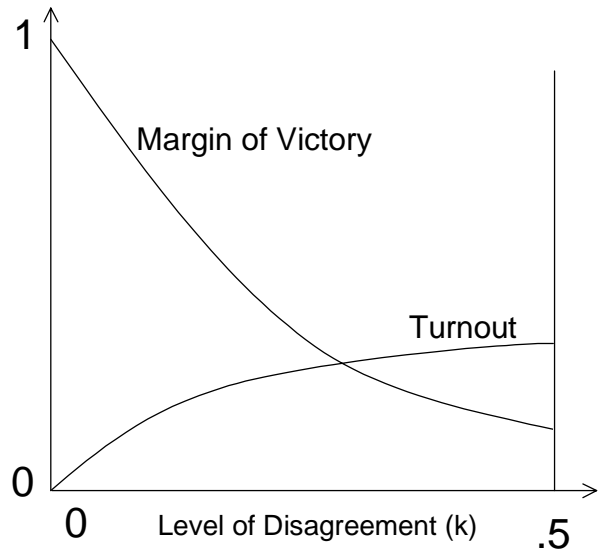

The intuition behind property 3 is as follows: When one group is an overwhelming majority then this group can win the election with high probability if only a small fraction of this group votes. So, turnout will be small and the margin of victory large. When the groups are of equal size then the margin of victory is small and turnout is large because no group can ensure victory at a low turnout level.

It is useful to contrast the properties of our model with the results that would be obtained in a purely decision theoretic model along the lines of Riker and Ordeshook (1968). In such a model turnout is determined by the fraction of the electorate with cost to vote less than $D$. So, the fraction of the majority and the minority who vote is given by $\min \left\{\frac{2 D}{\bar{c}}, 1\right\}$. Hence, unlike our model, the expected 
turnout of the minority is identical to the majority. Moreover, changing the level of disagreement would not change expected turnout in Riker and Ordeshook (1968).

Property 4. Expected turnout and margin of victory are increasing in the importance of election $(w)$ and decreasing in the average voting cost $(\bar{c})$.

The property that expected turnout increases with the important of the election is consistent with the evidence that turnout is higher for presidential elections than state elections (see Teixeira (1987)). The margin of victory is also increasing in the importance of the election. The intuition for this result is as follows: When the importance of the election is very high then both groups participate at maximum levels. Hence, the margin of victory is determined by the size of the groups. When the importance of the election decreases, turnout also decreases. This decreases the chances that the candidate favored by the majority will win (hence, decrease the expected margin of victory) because the fraction of the minority who vote is higher than the majority.

The inverse relationship shown in Figure 2 obtains as the level of disagreement changes. However, Property 4 shows that changes in the importance of the election changes then our model can produce a positive correlation between margin of victory and turnout.

Property 4 contrasts with the results in the decision theoretic literature. In Riker and Ordeshook neither turnout nor expected margin of victory change when the importance of the election change. Moreover, the expected margin of victory is also constant as expected voting cost changes. In our model, both the expected turnout and margin of victory increase in the importance of the election and decrease as expected voting cost changes. Property 4 is illustrated in Figure 3 below. 
Figure 3. Turnout and margin of victory as a function of the importance of the election.

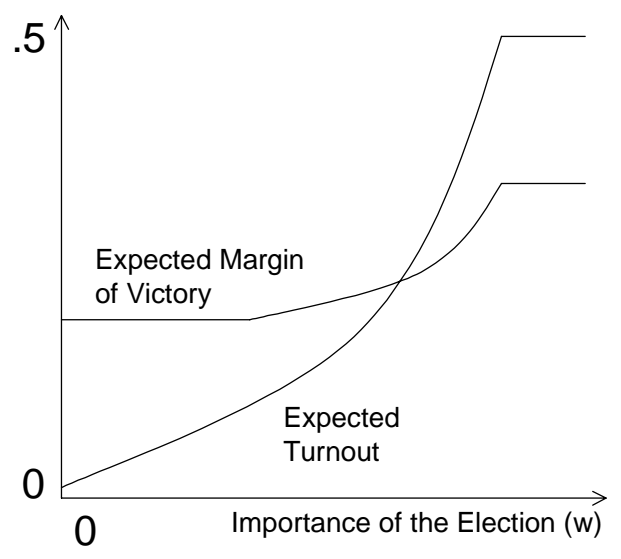

Property 5. Expected turnout is weakly increasing in the payoff for doing one's part $(D)$ while the expected margin of victory is weakly decreasing in $D$.

The comparative statics results on $D$ require us to consider the case in which $D<\frac{\bar{c}}{2}$. Property 5 shows an inverse correlation between the expected margin of victory and turnout as $D$ changes. In the decision-theoretic literature the $D$ term and the expected cost to vote $(\bar{c})$ are somewhat interchangeable since any changes in behavior depend only on the ratio $\frac{D}{\bar{c}}$. In our setting these two variables have distinct effects. Recall from figure 3 that the expected margin of victory and turnout decrease in $\bar{c}$. Property 5 is illustrated in figure 4 below. 
Figure 4. Turnout and margin of victory as a function of the payoff for doing one's part.

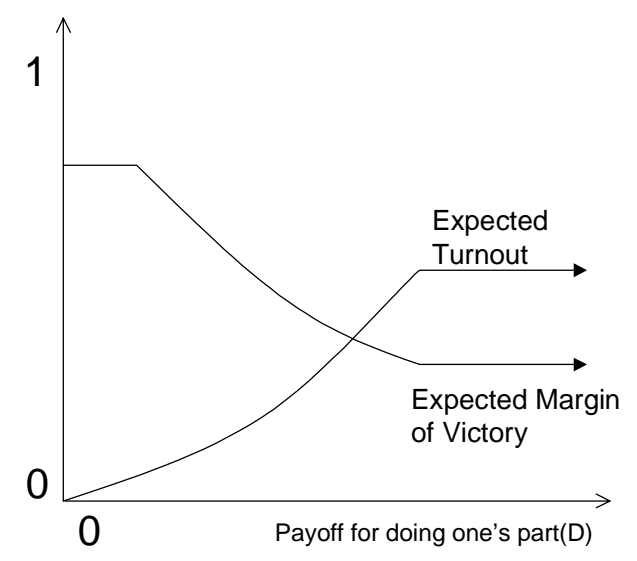

To provide an intuition for the result that the expected margin of victory is decreasing in $D$ consider the case in which $D$ is very small relative to $\bar{c}$. Then, the equilibrium is at a corner, i.e. $\sigma_{1}^{*}=\sigma_{2}^{*}=\frac{D}{2 \bar{c}}$. Only ethical agents with low costs vote. It would not be optimal for either player to reduce turnout because the reduction in total voting cost would be small. As $D$ increases, turnout increases. At some point, we move to a semi-corner equilibrium. Then, the fraction of the minority who vote is no longer equal to the fraction of the majority who vote. The fraction of the minority who votes becomes greater than the fraction of the majority who votes. This decreases the chances that the candidate supported by majority wins. It also decreases the expected margin of victory. 


\section{Existence and Uniqueness of Equilibrium}

In general, a pure strategy equilibrium may not exist in 2PG. For example, assume that fraction of the population in each group and the fractions of ethicals within the group is fixed, i.e., $\tilde{k}=k, \widetilde{q}_{1}=q_{1}$ and $\widetilde{q}_{2}=q_{2}$ where $k q_{1}>(1-k) q_{2}$. Then it is easy to see that an equilibrium in pure strategies does not exist. The problem is as follows: assume that the election is tied (i.e., $\sigma_{1} k q_{1}=\sigma_{2}(1-k) q_{2}$ ). Then, each candidate wins the election with equal probability and $\sigma_{2}<\sigma_{1} \leq 1$. So, there is an incentive for player 2 to slightly increase turnout and win the election. On the other hand, if the election is not tied then the winning player has an incentive to slightly decrease turnout so that it will still win, but at a smaller cost.

The absence of a pure strategy equilibrium in $2 \mathrm{PG}$ is especially problematic because mixed strategies in $2 \mathrm{PG}$ do not correspond to a consistent profile in the underlying game. A non-degenerate mixed strategy, for player $i$, in $2 \mathrm{PG}$ is a random variable distributed over $\left(0, \sigma^{u}\right]$ that does not put all weight on a single point. To see the problem of mapping a non-degenerate mixed strategy in $2 \mathrm{PG}$ into a consistent behavior profile first note that we don't consider rules in which agents randomize. Assume for the moment that we did allow rules that specify, for each agent, an independent random variable over the set of actions. In our model with a continuum of agents independent randomizations by each agent are equivalent to a rule with no randomization. Moreover, a rule in which a measurable set of agents actions are selected at random would not be maximally ranked. Consider a rule that requires a positive measure set of type $i$ agents to randomize between voting and abstaining with probabilities strictly between zero and one. Next, consider an alternative rule which is different only for these types. 
Instead of randomizing, the low cost types vote and the high cost types abstain, with the cut-off point chosen so that the expected vote share is identical in both cases. It follows that, fixing the behavior of the other types, the probabilities of winning for each candidate are unchanged but the expected social cost of voting is strictly smaller under the alternative rule.

An alternative way to generate mixed strategy-like behavior would be to allow agents of the same type to choose different rules. For example, assume that for a given $\sigma_{2}$ there exist two possible values $\sigma_{1}^{A}$ and $\sigma_{1}^{B}$ that maximize $\Pi_{1}$. This is equivalent to saying that there are two rules (defined by the cut-off points $c_{1}\left(\sigma_{1}^{A}\right)$ and $\left.c_{1}\left(\sigma_{1}^{B}\right)\right)$ that maximize $R_{1}$, under the assumption that all type 1 agents accept this rule. If some type 1 agents were to choose one rule and the rest chooses the other then each type 1 agent would have chosen a maximally ranked rule given the behavior of type 2 agents. However, the behavior generated among all type 1 agents would be inconsistent with either rule. Type 1 agents would be evaluating rules under false assumptions. Part 2 of the consistency definition requires that all agents with the same type behave as if they had chosen the same rule to avoid this problem.

Finally, a mixed strategy in 2PG could be mapped into a (more broadly defined) rule that uses a private correlating device for some group. All types in each group would accept the rule announced by the correlating device. However, it would be necessary that only types in the group observe the realization of their correlating device and that agents in the other group cannot infer the realization of this device. The existence of such private correlating device is problematic in the context of a large election.

Given the problems associated with mixed strategies we now identify sufficient 
conditions for the existence and uniqueness of a pure strategy equilibrium in 2PG. Consequently, we show that a consistent behavior profile exists in a class of elections. All proofs for the results in this section are available from the authors upon request.

A sufficient condition to guarantee the existence of an equilibrium in pure strategies is that the payoff functions for both players are strictly concave. ${ }^{10}$ Fortunately, it turns out that the negative expected aggregate voting cost functions are strictly concave.

Lemma 1. The function $C_{i}:\left(0, \sigma^{u}\right] \rightarrow \Re_{+}$is strictly increasing, twice continuously differentiable and strictly convex.

The intuition behind this result is that turnout increases as the cut-point for voting increases. The marginal cost increases as the cut-point increases because higher cost types are required to vote. So, marginal cost increases with turnout.

The expected probability of winning functions for each candidate are concave if the random variables

$$
\tilde{r} \equiv \frac{(1-\tilde{k}) \tilde{q}_{2}}{\tilde{k} \tilde{q}_{1}} \text { and } \frac{1}{\tilde{r}}=\frac{\tilde{k} \tilde{q}_{1}}{(1-\tilde{k}) \tilde{q}_{2}}
$$

are concave as defined below.

Definition 2. A random variable, $\tilde{x}$, that takes values on a subset of $\Re_{+}$, is concave if the cumulative density function $F_{\tilde{x}}(x)$ is a concave function of $x$ on $\Re_{+}$.

\footnotetext{
${ }^{10} \mathrm{~A}$ technical difficulty with this argument is that the best response functions are defined in a non-compact set $\left(0, \sigma_{i}^{u}\right]$. Details on how we solve this problem is available upon request.
} 
Any random variable on $\Re_{+}$whose density function is non-increasing is concave. For example, the uniform distribution over $(0,1)$ is concave. We can show that the random variables $\tilde{r}$ and $\frac{1}{\tilde{r}}$ are concave if assumption $A$ below is satisfied.

Assumption A: Let $\tilde{k}, \tilde{q}_{1}$ and $\tilde{q}_{2}$ be independent random variables. At least one of the pairs $\left(\tilde{q}_{1}, \tilde{q}_{2}\right),\left(\tilde{k}, \tilde{q}_{2}\right),\left(1-\tilde{k}, \tilde{q}_{1}\right)$ is such that both random variables are concave.

Clearly, assumption $A$ is satisfied if, as assumed in section $4, \tilde{k}$ is deterministic while $\tilde{q}_{1}$ and $\tilde{q}_{2}$ are independent and uniformly distributed over $(0,1)$.

Proposition 3. Under assumption $A$, a pure strategy equilibrium exists in $2 P G$ and is unique.

The uniqueness proof is involved and we cannot offer a simple intuition. Assumption $A$ is sufficient but not necessary to guarantee existence of a pure strategy equilibrium in $2 \mathrm{PG}$. For example, assume that $\tilde{k}=0.5, \tilde{q}_{1}=\tilde{q}_{2}=1$ and $\tilde{c} \sim U(0$, 1) then $\sigma_{1}^{*}=\sigma_{2}^{*}=1$ is an equilibrium. A pure strategy equilibrium can be obtained under weaker assumptions than assumption $A$. The assumption that $\tilde{q}_{1}$ and $\tilde{q}_{2}$ are independent can be relaxed. It suffices that conditional on $\tilde{q}_{j}=q_{j}$, $j \neq i, \tilde{q}_{i}$ is concave. The concavity assumption can also be relaxed. It would suffice to assume that the density function of $\tilde{q}_{i}, i \in\{1,2\}$, is single peaked. ${ }^{11}$ We do not demonstrate existence under these assumptions because the proof would be more involved and not as elegant as in the case of concave random variables. Moreover, $\tilde{q}_{i}, i \in\{1,2\}$, is concave in our main examples.

If the only source of uncertainty in the model was over the size of the groups $(\tilde{k})$ then $\tilde{r}$ and $\frac{1}{\tilde{r}}$ would not be concave for all $k \in(0,1)$. It is useful to have

\footnotetext{
${ }^{11}$ We would also require that $c^{l}=0$.
} 
at least two sources of uncertainty in order to ensure existence of equilibrium in $2 \mathrm{PG}$. This is the rationale for introducing uncertainty over the fraction of ethical agents in each group.

\section{General Comparative Statics}

In appendix $B$, we investigate the extent to which properties of section 4 hold when the assumptions of uniform distributions for voting costs and fractions of ethical agents are relaxed. We summarize our findings below.

Property 1 can be generalized. The fraction of voters in the minority group who participate in the election is greater than the fraction in the majority, but the majority has a higher chance of winning the election. ${ }^{12}$ Property 2 can also be generalized. Expected turnout goes to zero as the level of disagreement vanishes. Consistently with properties 3 and 4, we show that, under assumption $A$, expected turnout is increasing in the importance of the election and the expected margin of victory is decreasing on the level of disagreement.

Not every aspect of the example in section 4 generalizes. We provide an example in which expected turnout decreases as the disagreement level increases from a point to another. However, expected turnout can not monotonically decrease in the whole range of disagreement levels because it approaches zero when the level of disagreement vanishes.

\footnotetext{
${ }^{12}$ Note that this result depends on several assumptions. First, the importance of the election is equal for both groups. Second, agents in both groups care about aggregate voting costs as opposed to only caring about the voting cost of the members of their group.
} 


\section{Unifying the literature}

As we showed above, the model we analyze here is different from decision theoretic models e.g., Riker and Ordershook (1968). However, we can subsume their model as a special case. If the cost function $\vartheta$ in the agents' preferences is constant or increasing then agents should always vote, no matter the cost. In that case, just as in Riker and Ordeshook, agents will vote for their preferred candidate if and only if the payoff for doing their part is greater than their cost to vote. We can also formalize Harsanyi's (1992) model by assuming that all agents are utilitarians and that the level of disagreement is zero.

Models by Morton (1991), Nalebuff and Shachar (1999), and Ulhaner (1989) assume that elites provide incentives for group members to vote. It is easy to see that our 2PG game corresponds perfectly to a game between two elites who can increase participation among their supporters at some cost. However, the elite based models do not make the underlying mechanism generating turnout explicit. Perhaps elites make the voting costs of a part of the electorate negative. Naturally, in that case there would be no "paradox of voting." Our view is that payments to voters either directly or through social pressure are not sufficient to explain the full extent of turnout. As Nalebuff and Shachar (1999) note, an important component of the power of elites comes from their ability to persuade members of their group to vote. The difficulty is that without changing the costs to vote, standard rational actor models cannot explain how persuasion can motivate participation. Our model can provide the microfoundations for persuasive elites. There is a potential role for persuasion in our model because credible information about the importance of the election may increase turnout. Elites might be added to our 
model by introducing a stage prior to the election in which elites communicate with voters. Given that the interests of elites are not necessarily aligned with the interests of the electorate, such an extension is worth exploring.

\section{Conclusion}

We have developed a methodology in which agents in a game have actions they should take that are determined endogenously and influence payoffs. The application of this methodology to the problem of turnout in large elections provides testable implications and predicts variations in expected turnout and margin of victory as a function of costs to vote, level of disagreement within the society, importance of the election and agents' incentives to do their part. Coate and Conlin (2002) have structurally estimated our model and compared it to alternative models of turnout, with encouraging results.

Our methodology might usefully be applied to a variety of large voting games. For example, game-theoretic models of multi-candidate elections typically suffer from a multiplicity of equilibria. Perhaps our solution concept can provide more precise predictions about outcomes. Another potential application is large elections with asymmetrically informed voters. In game-theoretic models (see for example Feddersen and Pesendorfer 1999) voting behavior is determined as a consequence of voter beliefs in the unlikely event a vote is pivotal. Our approach may provide more compelling microfoundations for strategic voting behavior in such settings. We believe that the methodology developed here can be applied in a variety of settings outside the realm of large elections as well. 


\section{Appendix A:}

Proof of proposition 1 Without loss generality, we restrict attention to the case $D>c^{u}$. The proof for the case in which $D \leqslant c^{u}$ is analogous. Assume, by contradiction, that there is a strictly positive set of $\operatorname{costs} C$ such that $e_{i}^{*}(c)=\{$ vote for $j\}$ if $c \in C, j \neq i$. Consider an alternative rule in which agents who are required to vote for $j$ are instead required to vote for $i$. This rule would be ranked higher by type $i$ than $e_{i}^{*}$ because the expected voting cost is the same and the chances that $i$ wins the election is increased. Therefore, almost surely, $e_{i}^{*}(c)=\{$ vote for $i$ or abstain\}.

Let $c_{i}^{*}$ be the largest cost in $\left[c^{l}, c^{u}\right]$ such that for almost every cost $c \leqslant c_{i}^{*}$, $e_{i}^{*}(c)=\{$ vote for $i\}$. The proof is clearly complete if $c_{i}^{*}=c^{u}$. So, consider the case $c_{i}^{*}<c^{u}$. The proof would also be complete if $e_{i}^{*}(c)=\{$ abstain $\}$ for almost all costs $c>c_{i}^{*}$. So, consider a strictly positive measure set of costs $\bar{C} \subseteq\left(c_{i}^{*}, c^{u}\right]$ such that $e_{i}^{*}(c)=\{$ vote for $i\}$ if $c \in \bar{C}$. There exists $\bar{c}_{i}>c_{i}^{*}$ such that $e_{i}^{*}(c)=\{$ vote for $i\}$ if $c \in \bar{C} \cap\left(\bar{c}_{i}, c^{u}\right]$. By the definition of $c_{i}^{*}$ there is a strictly positive measure set of costs $\ddot{C}$ in $\left(c_{i}^{*}, \bar{c}_{i}\right)$ such that $e_{i}^{*}(c)=\{$ abstain $\}$ if $c \in \ddot{C}$. Pick a subset of $\ddot{C}$ and $\bar{C}$ $\bigcap\left(\bar{c}_{i}, c^{u}\right]$ with the same strictly positive measure. Consider an alternative rule in which the required actions for the costs in $\ddot{C}$ and $\bar{C} \bigcap\left(\bar{c}_{i}, c^{u}\right]$ are exchanged, but is otherwise identical to $e_{i}^{*}$. This rule would have a higher ranking than $e_{i}^{*}$ because the expected voting cost is reduced and the chance that $i$ wins the election is the same. This contradicts the definition of $e_{i}^{*}$. 


\section{Appendix B: General Comparative Statics}

In this section, we examine the generality of the properties of the election presented in section 4. To simplify the comparisons between the these results and the ones presented in section 4 we assume that the density of the costs of voting are identical for both groups; that the fraction of agents in group $1 \tilde{k}$ is a constant $k$; and that the average fraction of ethical agents in each group is the same and equal to $q \in(0,1)$.

Proposition 4. The minority turnout is greater than the majority. If $k<0.5$ then $\sigma_{1}^{*} \geqslant \sigma_{2}^{*}$.

Proof : The cost functions can be written as follows: $C_{1}\left(\sigma_{1}\right)=q k C\left(\sigma_{1}\right)$ and $C_{2}\left(\sigma_{2}\right)=q(1-k) C\left(\sigma_{2}\right)$. The proposition is clearly true if $\sigma_{1}^{*}=\sigma_{2}^{*}=\sigma^{u}$ or if $\sigma_{1}^{*}=\sigma^{u}$ and $\sigma_{2}^{*}<\sigma^{u}$. However, $\sigma_{1}^{*}<\sigma_{1}^{u}$ and $\sigma_{2}^{*}=\sigma_{1}^{u}$ cannot be an equilibrium because then

$$
k C^{\prime}\left(\sigma^{u}\right) \geqslant k C^{\prime}\left(\sigma_{1}^{*}\right) \geqslant \frac{\sigma^{u}}{\sigma_{1}^{*}}(1-k) C^{\prime}\left(\sigma^{u}\right) \geqslant(1-k) C^{\prime}\left(\sigma^{u}\right)>k C^{\prime}\left(\sigma^{u}\right) .
$$

If $\left(\sigma_{1}^{*}, \sigma_{2}^{*}\right)$ is an interior equilibrium then

$$
\frac{\sigma_{2}^{*}}{\sigma_{1}^{*}}=\frac{k}{1-k} \frac{C^{\prime}\left(\sigma_{1}^{*}\right)}{C^{\prime}\left(\sigma_{2}^{*}\right)} \Rightarrow \frac{C^{\prime}\left(\sigma_{2}^{*}\right) \sigma_{2}^{*}}{C^{\prime}\left(\sigma_{1}^{*}\right) \sigma_{1}^{*}}=\frac{k}{1-k} \leqslant 1 \Rightarrow \sigma_{2}^{*} \leqslant \sigma_{1}^{*} .
$$

Proposition 5. The majority has a higher chance of winning the election than the minority. If $k<0.5$ then $k \sigma_{1}^{*} \leqslant(1-k) \sigma_{2}^{*}$. 
Proof : The result is clearly true if $\sigma_{1}^{*}=\sigma_{2}^{*}=\sigma^{u}$. If $\sigma_{1}^{*}=\sigma^{u}$ and $\sigma_{2}^{*}<\sigma^{u}$ is an equilibrium then

$$
\frac{\sigma_{2}^{*}}{\sigma^{u}} \geqslant \frac{k}{1-k} \frac{C^{\prime}\left(\sigma^{u}\right)}{C^{\prime}\left(\sigma_{2}^{*}\right)} \Rightarrow(1-k) \sigma_{2}^{*} \geqslant k \frac{C^{\prime}\left(\sigma^{u}\right) \sigma^{u}}{C^{\prime}\left(\sigma_{2}^{*}\right)} \geqslant k \sigma^{u} .
$$

If $\left(\sigma_{1}^{*}, \sigma_{2}^{*}\right)$ is an interior equilibrium then

$$
\frac{\sigma_{2}^{*}}{\sigma_{1}^{*}}=\frac{k}{1-k} \frac{C^{\prime}\left(\sigma_{1}^{*}\right)}{C^{\prime}\left(\sigma_{2}^{*}\right)} \Rightarrow \frac{(1-k) \sigma_{2}^{*}}{k \sigma_{1}^{*}}=\frac{C^{\prime}\left(\sigma_{1}^{*}\right)}{C^{\prime}\left(\sigma_{2}^{*}\right)} \geqslant 1
$$

Proposition 6. Expected turnout goes to zero as one of the groups become an overwhelming majority. $T$ goes to 0 as $k$ goes to 0 .

Proof : Assume, by contradiction, that there exists $\varepsilon>0$ and subsequence such that $\sigma_{2}^{*} \geqslant \varepsilon$ as $k$ goes to 0 . Then, 2 wins the election with arbitrarily high probability no matter what player 1 does. If, instead player 2 votes at $0.5 \sigma_{2}^{*}$ then 2 will also win the election with arbitrarily high probability. The difference in benefits is arbitrarily small, but the difference in costs $\left(C_{2}\left(\sigma_{2}^{*}\right)-C_{2}\left(0.5 \sigma_{2}^{*}\right)\right)$ is bounded away from zero (greater than $C_{2}^{\prime}(\varepsilon) 0.5 \varepsilon$ ). A contradiction.

Proposition 7. Under assumption A, expected turnout is non-decreasing in the importance of the election $(w) .{ }^{13}$

\footnotetext{
${ }^{13}$ An alternative version of this result which does not require assumption A but places some restrictions on the distribution of cost (e.g. a uniform distribution) is available from the authors upon request.
} 
Proof : Let $h\left(\sigma_{1}, \sigma_{2}\right)=\vartheta^{\prime}\left(C_{1}\left(\sigma_{1}\right)+C_{2}\left(\sigma_{2}\right)\right)$. We will use this notation from here on. Assume without loss of generality that $k \leqslant 0.5$ (the proof for $k \geqslant 0.5$ is analogous). So, there is no semi-corner equilibrium in which $\sigma_{2}=\sigma^{u}$. Assume that $\left(\sigma^{u}, \sigma^{u}\right)$ is an equilibrium for some $\bar{w}$. Then,

$$
\bar{w} f_{\frac{(1-\tilde{k}) \tilde{q}_{2}}{\tilde{\tilde{q}} \tilde{q}_{1}}}(1) \frac{1}{\sigma^{u}} \geqslant h\left(\sigma^{u}, \sigma^{u}\right) \cdot C_{1}^{\prime}\left(\sigma^{u}\right) \text { and } \bar{w} f_{\frac{\tilde{k} \tilde{q}_{1}}{(1-\tilde{k}) \tilde{q}_{2}}}(1) \frac{1}{\sigma^{u}} \geqslant h\left(\sigma^{u}, \sigma^{u}\right) \cdot C_{2}^{\prime}\left(\sigma^{u}\right) .
$$

So, $\left(\sigma^{u}, \sigma^{u}\right)$ is an equilibrium for all $w \geqslant \bar{w}$. With some abuse of notation, let $\bar{w}$ be the smallest $w$ such that $\left(\sigma^{u}, \sigma^{u}\right)$ is an equilibrium. Assume that $\dot{\sigma}_{1}=\sigma^{u}$, $\dot{\sigma}_{2}<\sigma^{u}$ is a semi-corner equilibrium at $\dot{w}<\bar{w}$. We now show that the equilibrium at $\ddot{w}, \dot{w}<\ddot{w}<\bar{w}$ is also a semi-corner equilibrium. Note that the function

$$
j\left(\sigma_{2}\right) \equiv \frac{\sigma^{u} \cdot h\left(\sigma^{u}, \sigma_{2}\right) \cdot C_{2}^{\prime}\left(\sigma_{2}\right)}{f_{\frac{\tilde{k} \tilde{q}_{1}}{(1-\hat{\tilde{k}}) \tilde{q}_{2}}}\left(\frac{\sigma_{2}}{\sigma^{u}}\right)}
$$

is increasing and continuous in $\sigma_{2}$. Therefore, if $j\left(\sigma^{u}\right)=\bar{w}$ and $j\left(\dot{\sigma}_{2}\right)=\dot{w}$ then $j\left(\sigma_{2}\right)=\ddot{w}$ has a solution $\ddot{\sigma}_{2}$ such that $\sigma^{u}>\ddot{\sigma}_{2}>\dot{\sigma}_{2}$. By definition,

$$
\ddot{w} f_{\frac{(1-\tilde{k}) \tilde{q}_{2}}{\tilde{\kappa} \tilde{q}_{1}}}\left(\frac{\sigma^{u}}{\ddot{\sigma}_{2}}\right) \frac{1}{h\left(\sigma^{u}, \ddot{\sigma}_{2}\right) \cdot \ddot{\sigma}_{2}}=C_{2}^{\prime}\left(\ddot{\sigma}_{2}\right) \frac{\ddot{\sigma}_{2}}{\sigma^{u}}>\frac{C_{2}^{\prime}\left(\dot{\sigma}_{2}\right) \dot{\sigma}_{2}}{\sigma^{u}} \geqslant C_{1}^{\prime}\left(\sigma^{u}\right)
$$

where the last inequality comes from the fact that $\left(\sigma^{u}, \dot{\sigma}_{2}\right)$ is an equilibrium. Therefore, $\left(\sigma^{u}, \ddot{\sigma}_{2}\right)$ is an equilibrium at $\ddot{w}$. Hence, if $\left(\sigma^{u}, \dot{\sigma}_{2}\right)$ is a semi-corner equilibrium at $\dot{w}<\bar{w}$ then there exists a segment $[\tilde{w}, \bar{w})$ in which the equilibrium is semi-corner and expected turnout is non-decreasing in $w$.

Consider a segment $(0, \tilde{w})$ in which the equilibrium $\left(\sigma_{1}^{*}, \sigma_{2}^{*}\right)$ is interior. Then,

$$
\frac{\sigma_{2}^{*}}{\sigma_{1}^{*}}=\frac{C_{1}^{\prime}\left(\sigma_{1}^{*}\right)}{C_{2}^{\prime}\left(\sigma_{2}^{*}\right)}
$$


implies that $\left(\sigma_{1}^{*}, \sigma_{2}^{*}\right)$ are either both increasing or decreasing in $w \in(0, \tilde{w})$. Assume that both $\left(\sigma_{1}^{*}, \sigma_{2}^{*}\right)$ are decreasing in $w \in(0, \tilde{w})$. Note that

$$
\begin{aligned}
& w f_{\frac{\tilde{k} \tilde{q}_{1}}{(1-\tilde{k}) \tilde{q}_{2}}}\left(\frac{\sigma_{2}^{*}}{\sigma_{1}^{*}}\right)=\sigma_{1}^{*} \cdot h\left(\sigma_{1}^{*}, \sigma_{2}^{*}\right) \cdot C_{2}^{\prime}\left(\sigma_{2}^{*}\right) ; \\
& w f_{\frac{(1-\tilde{\tilde{k}}) \tilde{q}_{2}}{\tilde{k} \tilde{q}_{1}}}\left(\frac{\sigma_{1}^{*}}{\sigma_{2}^{*}}\right)=\sigma_{2}^{*} \cdot h\left(\sigma_{1}^{*}, \sigma_{2}^{*}\right) \cdot C_{1}^{\prime}\left(\sigma_{1}^{*}\right) .
\end{aligned}
$$

The first equation implies that $\frac{\sigma_{2}^{*}}{\sigma_{1}^{*}}$ is strictly decreasing in $w$ while the second would imply that $\frac{\sigma_{2}^{*}}{\sigma_{1}^{*}}$ is strictly increasing in $w$.

Proposition 8. Under assumption $A$, the expected margin of victory is decreasing in the level of disagreement $(k)$.

Proof: Assume without loss of generality that $k \leqslant 0.5$ (the proof for $k \geqslant 0.5$ is analogous). So, there is no semi-corner equilibrium in which $\sigma_{2}=\sigma^{u}$.

$$
\begin{gathered}
w f\left(\frac{\sigma_{2}}{\bar{k} \sigma_{1}}\right) \frac{1}{\bar{k} \sigma_{1}}-(1-k)\left(\frac{\bar{c}}{2}\right) \sigma_{2}=0 ; \text { if } \sigma_{2} \in(0,1) . \\
w f\left(\bar{k} \frac{\sigma_{1}}{\sigma^{u}}\right) \frac{\bar{k} \sigma_{1}}{\left(\sigma_{2}\right)^{2}}-(1-k)\left(\frac{\bar{c}}{2}\right) \sigma_{2}=0 ; \text { if } \sigma_{2} \in(0,1) .
\end{gathered}
$$

The expected margin of victory is a positive function of $m \equiv \frac{\sigma_{2}(1-k)}{\sigma_{1} k}$. If the equilibrium is a corner solution then $m$ is clearly decreasing in $k$. If the equilibrium is a semi-corner solution then $w f(m) \cdot \frac{1}{\sigma^{u}}=k h\left(\sigma^{u}, \sigma_{2}\right) C^{\prime}\left(\sigma_{2}\right)$, where $f$ is the density of $\frac{\tilde{q}_{1}}{\tilde{q}_{2}}$. The function $\frac{w f(m)}{\sigma^{u} h\left(\sigma^{u}, \sigma_{2}\right) C^{\prime}\left(\sigma_{2}\right)}$ is decreasing in $\sigma_{2}$. So, in a semi-corner equilibrium, $\sigma_{2}$ is decreasing in $k$ and, therefore, $m=\frac{\sigma_{2}(1-k)}{\sigma^{u} k}$ is decreasing in $k$.

Now consider an interior equilibrium. Assume, by contradiction, that as $k$ increases, $m$ increases or stays constant. Then, $\frac{\sigma_{2}}{\sigma_{1}}$ increases. However, in an 
interior equilibrium we have $\frac{\sigma_{2}}{\sigma_{1}} \frac{k}{(1-k)}=\frac{C^{\prime}\left(\sigma_{1}\right)}{C^{\prime}\left(\sigma_{2}\right)}$. So, $\frac{C^{\prime}\left(\sigma_{1}\right)}{C^{\prime}\left(\sigma_{2}\right)}$ increases. Hence, either both $\sigma_{1}$ and $\sigma_{2}$ increase or they both decrease. If they both increase a contradiction obtains because $f(m)$ decreases and $w f(m)=k \sigma_{1} h\left(\sigma_{1}, \sigma_{2}\right) C^{\prime}\left(\sigma_{2}\right)$. If they both decrease then a contradiction obtains because $f\left(\frac{1}{m}\right)$ increases and $w f\left(\frac{1}{m}\right)=(1-k) \sigma_{2} h\left(\sigma_{1}, \sigma_{2}\right) C^{\prime}\left(\sigma_{1}\right)$.

We now show that property 3 does not generalize and expected turnout may not increase monotonically on the level of disagreement.

Example 2. The fraction of ethical agents in each group, $\widetilde{q}_{1}$ and $\widetilde{q}_{2}$, are independent with density

$$
f(x)=\left\{\begin{array}{l}
4 \text { if } x \in(0,0.1) \\
\frac{2}{3} \text { if } x \in(0.1,1)
\end{array}\right.
$$

The level of disagreement is fixed $(\tilde{k}=k)$. Voting costs are given by $\tilde{c} \sim \frac{1}{2} U(0$, $1)$. The payoff $D$ is greater than $0.5, \vartheta(x)=x$ and $w=\frac{100}{44}$.

In this example, $q=\frac{7}{20}$. The density of $\frac{\tilde{q}_{1}}{\tilde{q}_{2}}$ and $\frac{\tilde{q}_{2}}{\tilde{q}_{1}}$ is given by

$$
\bar{f}(x)=\left\{\begin{array}{c}
1.4 \text { if } x \leqslant 0.1 \\
\frac{39}{135}+\frac{1}{90 x^{2}} \text { if } 0.1 \leqslant x \leqslant 1 \\
\frac{39}{135 x^{2}}+\frac{1}{90} \text { if } 1 \leqslant x \leqslant 10 \\
\frac{1.4}{x^{2}} \text { if } 10 \leqslant x .
\end{array}\right.
$$

Let $k=\frac{1}{11}$. Then, $\bar{k}=\frac{k}{1-k}=\frac{1}{10}$. Note that $\sigma_{1}^{*}=\sigma_{2}^{*}=1$ is an equilibrium because

$$
\frac{14}{44}=w \bar{f}\left(\frac{1}{\bar{k}}\right) \frac{1}{\bar{k}} \geqslant q(1-k)=\frac{14}{44} .
$$

Let $k=\frac{1}{10}$. Then, $\bar{k}=\frac{k}{1-k}=\frac{1}{9}$. So, $\sigma_{1}^{*}=\sigma_{2}^{*}=1$ is not an equilibrium because

$$
\frac{100}{44}\left(\frac{39}{135}+\frac{81}{90}\right) \frac{1}{9}=w \bar{f}\left(\frac{1}{\bar{k}}\right) \frac{1}{\bar{k}}<q(1-k)=\frac{7}{20}\left(1-\frac{1}{10}\right) .
$$


It follows that expected turnout decreases when $k$ increases from $\frac{1}{11}$ to $\frac{1}{10}$.

\section{References}

[1] Aldrich, J. "Rational Choice and Turnout," American Journal of Political Science, 37-1, 246-278.

[2] Andreoni, J. (1989) "Giving with Impure Altruism: Applications to Charity and Ricardian Equivalence," Journal of Political Economy, 97-6, 1447-1458.

[3] Becker, G. (1976) "Altruism, Egoism, and Genetic Fitness: Economics and Sociobiology," Journal of Economic Literature, 14-3, 817-826.

[4] Becker, G. (1977) "Altruism, Egoism, and Genetic Fitness: Economics and Sociobiology," Journal of Economic Literature" 14-3, 817-826

[5] Bergstrom, T. (1995) "On the Evolution of Altruistic Ethical Rules for Siblings," American Economic Review, 85-1, 58-81.

[6] Bernheim, D. and O. Stark (1988) "Altruism within the Family Reconsidered: Do Nice Guys Finish Last?" American Economic Review, 78-5, 1034-1045.

[7] Blais, A. (2000) To Vote or Not to Vote: The Merits and Limits of Rational Choice Theory. University of Pittsburgh Press.

[8] Coate, S. and M. Conlin (2002) "Voter Turnout: Theory and Evidence from Texas Liquor Referenda," NBER Working Paper No.w8720. 
[9] Green, D. and I. Shapiro (1996) Pathologies of Rational Choice Theory: A Critique of Applications in Political Science. New Haven: Yale Univ Press.

[10] Harsanyi, J. (1977) "Morality and the Theory of Rational Behavior." Social Research 44: 623-656.

[11] Harsanyi, J. (1980) "Rule Utilitarianism, Rights, Obligations and The Theory of Rational Behavior," Theory and Decision, 12, 115-33.

[12] Harsanyi, J. (1992) "Game and Decision Theory in Ethics," Handbook of Game Theory, vol 1, Edited by R. Aumann and S. Hart.

[13] Hirshleifer, J. (1985) "The Expanding Domain of Economics," American Economic Review, 75-6, 53-68.

[14] Kahneman, D., and A. Tversky (1979) "Prospect theory: An Analysis of Decision Under Risk," Econometrica, 47, 263-291.

[15] Feddersen, T. and W. Pesendorfer (1999) "Voting Behavior and Information Aggregation in Elections with Private Information," Econometrica, 65-5, 1029-1058.

[16] Franklin, M, R. Niemi and G. Whitten (1994) "Two Faces of Tactical Voting," British Journal of Political Science, 24, 549-57.

[17] Goodin, R. and K. Roberts (1975) "The Ethical Voter," American Journal of Political Science, 69, 1227-85.

[18] Margolis, H. (1982) Selfishness, Altruism and Rationality, Cambridge University Press. 
[19] Monroe, K. (1994)) "A Fat Lady in a Corset: Altruism and Social Theory," American Journal of Political Science, 38- 4, 861-893.

[20] Morton, R. (1991) "Groups in Rational Turnout Models," American Journal of Political Science, 35, 758-76.

[21] Nalebuff, N. and R. Shachar (1999) "Follow the Leader: Theory and Evidence on Political Participation," American Economic Review, 89-3, 525-47.

[22] Palfrey, T., and H. Rosenthal (1983) "A Strategic Calculus of Voting," Public Choice, 41-1, 7-53.

[23] Palfrey, T., and H. Rosenthal (1985) "Voter Participation and Strategic Uncertainty," American Political Science Review, 79-1, 62-78.

[24] Rabin, M. (1993) "Incorporating Fairness into Game Theory and Economics," American Economic Review, 83, 1281-1302.

[25] Riker, W., and P. Ordeshook (1968) "A Theory of the Calculus of Voting," American Political Science Review, 62, 25-42.

[26] Roemer, J. (1996) Theories of Distributive Justice. Harvard University Press.

[27] Samuelson, P. (1993) "Altruism as a Problem Involving Group versus Individual Selection in Economics and Biology," American Economic Review, Vol. 83, No. 2, Papers and Proceedings of the Hundred and Fifth Annual Meeting of the American Economic Association, 143-148.

[28] Sen, A. (1977) "Social Choice Theory: A Re-Examination," Econometrica, 45-1, 53-89. 
[29] Simon, H. (1955) "A Behavioral Model of Rational Choice," The Quarterly Journal of Economics, Vol. LXIX, February.

[30] Sugden, R. (1984) "Reciprocity: The Supply of Public Goods Through Voluntary Contributions," The Economic Journal, 94, 772-787.

[31] Teixeira, R. (1987) Why Americans Don't Vote: Turnout Decline in the United States: 1960-1984. New York Greenwood Press.

[32] Uhlaner, C. (1989) "Rational Turnout: The Neglected Role of Groups," American Journal of Political Science, 33-390-422. 